\title{
Study of Lapping and Polishing Performance on Lithium Niobate Single Crystals
}

\author{
Karim Ravilevich Muratov ${ }^{1}$, Timur Rizovich Ablyaz ${ }^{1, *}$, Evgeny Anatolevich Gashev ${ }^{1}$, Sarabjeet Singh Sidhu ${ }^{2} \mathbb{D}$ \\ and Evgeny Sergeevich Shlykov ${ }^{1}$ \\ 1 Mechanical Engineering Faculty, Perm National Research Polytechnic University, 614000 Perm, Russia; \\ karimur_80@mail.ru (K.R.M.); kot_ostrow@mail.ru (E.A.G.); kruspert@mail.ru (E.S.S.) \\ 2 Mechanical Engineering Department, Sardar Beant Singh State University (Formerly, Beant College of \\ Engineering and Technology), Gurdaspur 143521, India; sarabjeetsidhu@yahoo.com \\ * Correspondence: lowrider11-13-11@mail.ru
}

Citation: Muratov, K.R.; Ablyaz, T.R.; Gashev, E.A.; Sidhu, S.S.; Shlykov, E.S. Study of Lapping and Polishing Performance on Lithium Niobate Single Crystals. Materials 2021, 14, 4968. https://doi.org/10.3390/ ma14174968

Academic Editors: Wiesław A. Graboń and Thomas G. Mathia

Received: 14 July 2021

Accepted: 26 August 2021

Published: 31 August 2021

Publisher's Note: MDPI stays neutral with regard to jurisdictional claims in published maps and institutional affiliations.

Copyright: (c) 2021 by the authors. Licensee MDPI, Basel, Switzerland. This article is an open access article distributed under the terms and conditions of the Creative Commons Attribution (CC BY) license (https:/ / creativecommons.org/licenses/by/ $4.0 /)$.

\begin{abstract}
Recently, the range of crystal materials used in industrial microelectronics has significantly increased. Lithium niobate single crystals are most often used in integrated optics, due to the high values of optical and electro-optical coefficients. An integral-optical circuit based on a lithium niobate single crystal is a key element in the production of local high-precision fiber-optic gyroscopic devices used in civil and military aviation and marine technologies. In the process of production of an integral-optical circuit, the most labor-intensive operations are mechanical processing, such as lapping and polishing. Technological problems that arise while performing these operations are due to the physical and mechanical properties of the material, as well as target surface finish. This work shows the possibility to achieve the required surface quality of lithium niobate single crystal plates by mechanization of lapping and polishing process in this article.
\end{abstract}

Keywords: lithium niobate single crystal; abrasive lapping; polishing; surface roughness; deformed layer; tool wear; kinematics

\section{Introduction}

Recently, the range of crystal materials used in industrial microelectronics has significantly increased. One such material is lithium niobate single crystals (LNSC). In integrated optics, due to their high optical and electro-optical coefficient values, LNSC are most often used.

The main processes for obtaining accurate and clean surfaces in the production of optical components are finishing and lapping with abrasive powders in a free or fixed state. The deformation mechanism during abrasive processing of various optical materials is not the same and depends on their physical and mechanical properties [1-5]. For instance, while processing plastic based optical materials, the underlined material removal phenomenon is the removal of material in the form of thin layers, thus the appearance of plastic deformation in neighboring areas. However, while processing crystalline materials like germanium silicon and glass, the resulting microcracks penetrate to a certain depth from the surface and form a deformed subsurface layer [6,7]. Thus, the abrasive processing of plastic and crystalline/brittle materials differ significantly.

Many studies have been devoted to the research of the phenomena underlying the processes of abrasive processing of brittle optical materials. That most of the studies of abrasive dispersion are mainly carried out on glass material. In comparison to glass, most crystalline substances are much lower in mechanical properties. However, few have high strength and hardness, such as optical ceramics. The theory of the impact of free abrasive grains on the glass surface during its lapping is now generally accepted and has found its further development and refinement [6,8-13]. There are some differences in the operation of free and fixed abrasive grains which significantly influence the mechanism of action, 
the size of the deformed layer, and the micro relief of the processed surface $[9,14,15]$. In studies $[1,9,10,16]$, it was found that the ratio of the depth $F$ of the deformed and $h$ of the relief layers for various grades of optical glass and minerals with a similar brittle criterion remains almost constant: $F / h=K \approx 4$ (Figure 1 ).

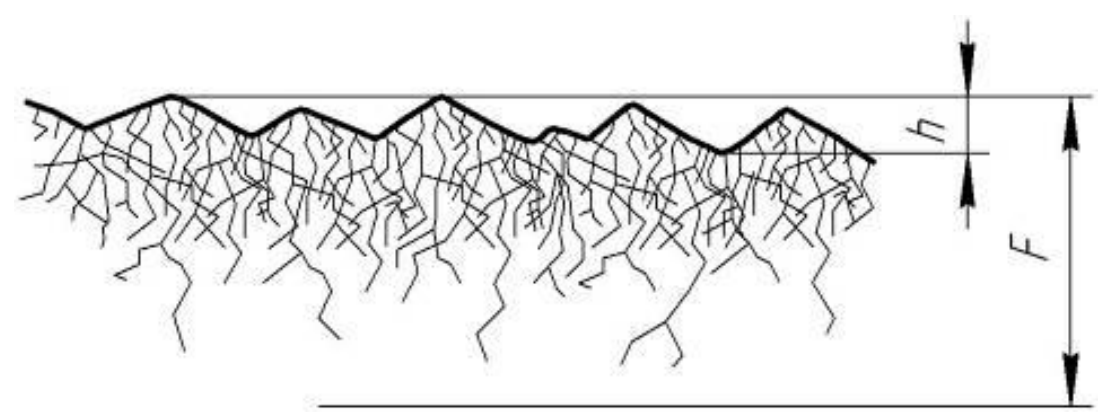

Figure 1. Structure of the deformed layer during lapping of brittle materials.

The size of the abrasive grain and its hardness have the most significant impact on the depth of the relief layer. In contrast, the hardness of the lapping material, pressure, and speed of finishing have a negligible impact. During the abrasive processing of crystalline materials, for example, single crystals of lithium fluoride and fluorite, the direction of cracks propagation and resulting strain differs from their direction in the glass and depends on their structure. Therefore, the microrelief of lapped surface of crystals is also related to the structure and the crystallographic direction in which the lapping is preformed [1,17].

The process of polishing brittle optical materials removes the deformed layer caused by lapping and surface texture whose defects are significantly less than the wavelength of light (about $1 / 4-1 / 8 \lambda$ ) also creates. As a result, the surface becomes transparent and perfectly smooth. After polishing, the deformed layer of crystalline materials is also heterogeneous and has a complex structure [10,11]. Even after chemical-mechanical polishing using ultra and nano-disperse diamond powders [11], the nano scratches of various types were detected on the processed surface: dash-point or dot-shaped, short and long, straight and arc-shaped. Below the relief zone area is an elastic-stressed, where surface structural defects may occur. The sum of these areas determines the depth of the deformed layer resulting from polishing.

The abrasive finishing of end surfaces of LNSC plates is not found in the literature so far. Hence, the question of the depth of the deformed layer and the features of precision processing of modern single-crystal materials, such as LNSC, remains almost unstudied. Fine-turning and polishing small areas in relation to the lapping area causes local tool wear. This affects the quality of the processed surface integrity. The uniform distribution of processing traces depends on the size of the surface to be processed and the tool, and development of technology for mechanized lapping and polishing of optical materials to ensure good quality.

In the present study, an attempt has been made to improve the efficiency of lapping and polishing by mechanizing the process of abrasive finishing of the ends LNSC plates. To achieve the same, the effect of the trajectory of tool on the quality of surface is investigated and quantified by the depth of the deformed layer. Furthermore, the possibility of achieving the required surface quality of the LNSC plates based on the mechanization of the polishing process is analyzed and quantitative and qualitative indicators are established.

\section{Material and Methods}

LNSC plates after diamond cutting were used as samples in experiments. The length dimensions of the workpiece is $36 \times 3.04 \times 12.84 \mathrm{~mm}(1 \times \mathrm{w} \times \mathrm{h})$. Processing of the end faces of the plates was carried out in packages of $4-5$ pieces in a special fixture on the finishing and polishing machine (Innovation 200R Twin, Remet, Italy). The necessary pressure on the tool-work surface contact was generated and changed by calibrated weights. 
The original raw and processed surfaces of the ends were evaluated visually and by optical microscopy (Olympus GX51 and CAM-MS-01. USB). Surface roughness parameters $\mathrm{Ra}, \mathrm{Rz}$, and Rmax were measured using a profilometer (Perthometer S2, Mahr GmbH, Esslingen am Neckar, Germany) and profilograms were recorded to assess of the microrelief. Measurements of the geometric form deviation of the processed LNSC plates and the tool were performed using form tester (MarForm MMQ 400, Mahr GmbH, Esslingen am Neckar, Germany). The productivity of the process (removal rate) was estimated by the value of linear removal of the material per time $Q(\mu \mathrm{m} / \mathrm{min})$. A vertical optical long-range meter IZV-2 (USSR) with a division of $1 \mu \mathrm{m}$ (Figure 2) was used to measure the amount of removal. This allowed rapid and minimal measuring effort for high accuracy.

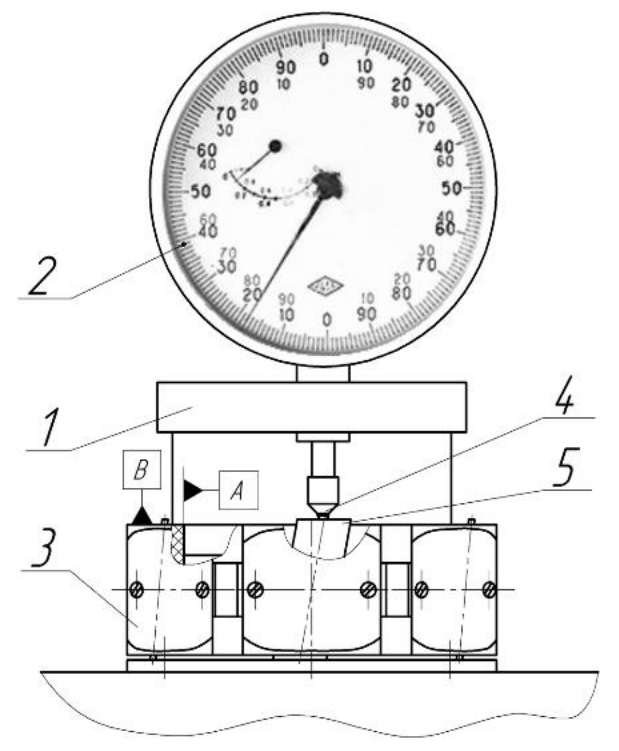

Figure 2. Device for measuring the amount of material removal: 1—case; 2 -indicator head; 3 fixture; 4-tip; 5-package of plates.

Indicator head 2 type 1MIG, GOST 9696-82 with a division of $1 \mu \mathrm{m}$, is fixed to the case device 1 . The device is based on the inner diameter $A$ and the ends surface $B$ of the case fixture 3.The tip 4 of the indicator 2 is oriented above the package of workpieces 5 and the measurement is carried out. The material's removal rate $\left(\Delta_{A m i d}, \mu \mathrm{m}\right)$ is defined as the difference between the indicator readings before and after processing. To assess of the depth of deformed layer of the plate ends, electron scanning microscopy was used (SEM Hitachi S-3400N, Japan). Before analyzing the samples in SEM, the surfaces of the same were coated with a layer of gold with a thickness of 1 nanometer. Figure 3 shows the installation scheme of sample (1) on table (2) in the working area of the microscope (1).This installation scheme provided the determination of the width and depth of the deformed layer. Setup scan parameters are an accelerating voltage- $20 \mathrm{kV}$, zoom from $\times 350$ to $\times 2500$, secondary electron mode, mode of electron backscattering.

Suspensions were prepared by mixing micro-powders made of white electrocorundum with a grain size of 24A M14, 24A M7, 24A M3 in deionized water lapping with a free abrasive. The concentration of the abrasive suspension by weight was $1 / 5$. The base suspension in comparative experiments was 24A M7.

Polishing was performed on suspensions based on deionized water and synthetic diamond micro-powders ASM with a grain size of $1 / 0.5$ and $0.5 / 0$. The concentration of the suspension by weight was kept $1 / 10$. The study of the lapping process using suspensions was carried out on round lappers (Russia) with a diameter of $200 \mathrm{~mm}$ made of optical glass grades K8 and LK5. Diamond lapping ASN 10/7-B3-01-2 and ASN 10/7-M2-01-2 were used for lapping with a fixed abrasive. Woven and non-woven hydrophobic materials were used as polishers in experiments were raincoat fabric (Bologna); nylon mesh with a 
thickness of $0.09 \mathrm{~mm}$, cell size $\sim 0.1 \mathrm{~mm}$; polyethylene wrap with a thickness of $0.14 \mathrm{~mm}$; raincoat fabric ( $80 \%$ polyamide, $20 \%$ polyurethane); awning synthetic fabric with a waterrepellent coating; VelTex Buehler polisher (Switzerland); MicrolapRemet polisher (Italy); synthetic leather SK-8.The durability of the lapping working surface was evaluated by the running time between edits. The durability of polishers was also estimated by the total operating time before the appearance of local physical wear on the work surface or before a significant decrease in the polishing ability and the formation of visible marks on the processing surface.

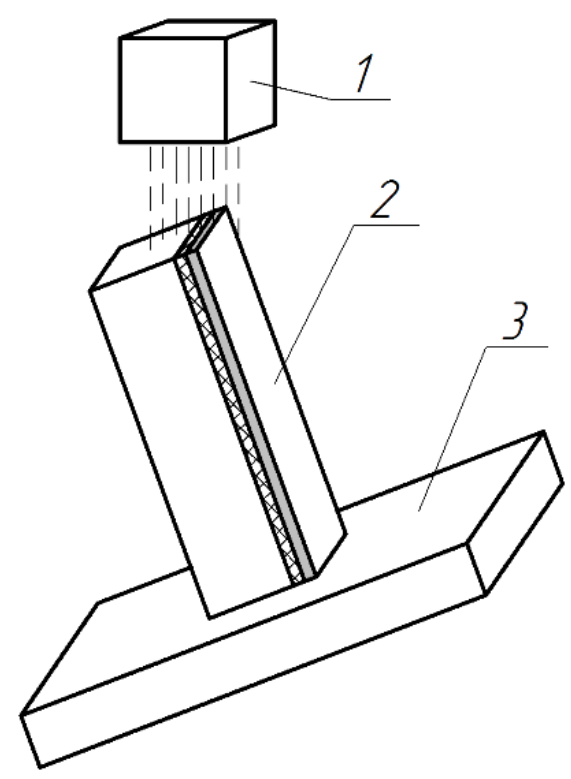

Figure 3. Installation of the sample during scanning: 1-detector; 2 -sample; 3 -the table.

\section{Results and Discussion}

Firstly, the influence of the type of trajectory on the lapped surface quality and the process productivity was determined. Machines with a raster and cycloidal trajectory movement, as well as rotational tool movement, were examined. A multi-position fixture was used to hold the workpieces and allow them to move along the lapping surface. Three sets of plates were installed in the position of the fixture. The size of the processed plates was $36 \times 3 \times 1 \mathrm{~mm}$. Two types of lapps were used in the experiments.

A glass disk made of optical glass of the $\mathrm{K} 8$ brand was used with a suspension of white 24A electrocorundum with a grain size of $\mathrm{M} 7 \mu \mathrm{m}$ in deionized water, with a concentration of 1/5. The lapp with the fixed abrasive ASN 10/7-B3-01-2 mark was used with water as a cutting fluid. The constant processing modes were: cutting speed $=0.18 \mathrm{~m} / \mathrm{s}$, pressure $\mathrm{P}=64 \mathrm{kPa}$ at a clamping force of $0.24 \mathrm{~N}$. Processing was performed for $1 \mathrm{~min}$. Table 1 shows the mean values of output processing parameters.

The processing performance was increased by $25-30 \%$ when the kinematics of the lapping motion becomes more complex with any finishing method, but the surface roughness does not change significantly. The dependence of the increasing debris during the trajectory was examined with optical microscopy of the plate ends before and after processing. The largest spalls/debris (with a depth of more than $45 \mu \mathrm{m}$ ) after processing with a fixed abrasive on the Raster 220 lapping machine was detected. As a result, machines with a tool working movement with alternating speeds were not recommended for lapping operations of such fragile materials as LNSC. 
Table 1. Kinematics influence on the parameters of the lapping process.

\begin{tabular}{|c|c|c|c|c|c|}
\hline Tool & Trajectory & Rotation & $\frac{\text { Cycloid }}{\gamma_{\gamma \gamma}^{\gamma}}$ & $\frac{\text { Rastr }}{2}$ & $\begin{array}{l}\text { Roughness and } \\
\text { Productivity }\end{array}$ \\
\hline \multirow{4}{*}{\multicolumn{2}{|c|}{$\begin{array}{l}\text { Free abrasive } \\
\text { 24A M7 }\end{array}$}} & 0.173 & 0.183 & 0.170 & $\mathrm{Ra}, \mu \mathrm{m}$ \\
\hline & & 1.322 & 1.375 & 1.215 & $\mathrm{Rz}, \mu \mathrm{m}$ \\
\hline & & 1.491 & 1.556 & 1.555 & $\operatorname{Rmax}, \mu \mathrm{m}$ \\
\hline & & 7.1 & 9.4 & 10.4 & $Q, \mu \mathrm{m} / \mathrm{min}$ \\
\hline \multirow{4}{*}{\multicolumn{2}{|c|}{$\begin{array}{l}\text { Fixed abrasive } \\
\text { ASN } 10 / 7\end{array}$}} & 0.078 & 0.074 & 0.088 & $\mathrm{Ra}, \mu \mathrm{m}$ \\
\hline & & 0.674 & 0.553 & 0.694 & $\mathrm{Rz}, \mu \mathrm{m}$ \\
\hline & & 0.852 & 0.669 & 0.809 & $R \max , \mu \mathrm{m}$ \\
\hline & & 8.4 & 11.4 & 12 & $Q, \mu \mathrm{m} / \mathrm{min}$ \\
\hline
\end{tabular}

The satisfactory results on the quality of LNSC plates were obtained during lapping on a machine with a rotational lapp movement. No large spalls were detected, regardless of the type of tool used. According to technical requirements, the roughness of the processed surface should not exceed $0.003 \mu \mathrm{m}$ in the Ra parameter, while individual scratches on the surface and edges are not allowed. The main requirements for the plates fixture and parameters set up in the lapping machine were formulated by taking into account the high brittleness of materials and a small area of the processed surface:

(1) To increase the area of the processed surface, it is necessary to process the plates in a set of 3-5 pieces;

(2) To self-positioning of the fixture with workpieces on the lapp surface, the number of sets must be at least three;

(3) Information about the acceptable deformation of LNSC plates was necessary for the development of their mechanical fixing;

(4) The use of standard pressure spring or pneumatic mechanisms of lapping machines to create working pressure was not allowed due to the high brittleness of the material;

(5) Processing with the workpieces beyond the lapp was not allowed to avoid the appearance of spalls on the processed surface and edges;

(6) Setting parameters must ensure minimum possible uniform wear of the lapp;

(7) Local wear of lapp should not exceed $20 \mu \mathrm{m}$ so as to reduce the probability of the edge spalling and processed surface damage by fragments of the material.

A full factorial experiment was completed based on the stated requirements for fixture and setting parameters. The regression equation was obtained and allows us to estimate the value of local lapp wear

$$
y=8.389+5.461 \cdot x_{1}+0.54 \cdot x_{2}+1.313 \cdot x_{3}
$$

The values of factors are used for decoding

$x_{1}=2 \cdot\left(r-r_{0}\right) /\left(r_{\max }-r_{\min }\right), \quad x_{2}=2 \cdot\left(n-n_{0}\right) /\left(n_{\max }-n_{\min }\right), \quad x_{3}=\left(2 \cdot\left(r-r_{0}\right) /\left(r_{\max }-r_{\min }\right)\right)^{2}$,

where $r$-radius of the workpieces location in the fixture, $\mathrm{mm}$ (limits: $r_{\min }=25 \mathrm{~mm}, r_{\max }=$ $44.5 \mathrm{~mm}) ; n$-frequency of lapprotation, rpm (limits: $\left.n_{\min }=42 \mathrm{rpm}, n_{\max }=150 \mathrm{rpm}\right) ; r_{0}$ $=\left(r_{\max }+r_{\min }\right) / 2$ - the main factor level, $\mathrm{mm} ; n_{0}=\left(n_{\max }+n_{\min }\right) / 2$ - the main factor level, rpm.

Substituting (2) in (1) we get a mathematical dependence

$$
\Delta h=55+0.06 \cdot r^{2}-3.61 \cdot r+0.01 \cdot n,
$$

where $\Delta h$-working tool surface wear, $\mu \mathrm{m}$. 
Equation (3) is reduced to a dimensionless form for ease of use

$$
\Delta h / h_{\text {alw }}=1100+1450 \cdot\left(r / r_{0}\right)^{2}-2509 \cdot r / r_{0}+19.2 \cdot n / n_{0}
$$

where $h_{a l w}=20$ - the value of allowable wear, $\mu \mathrm{m}$.

Dependence of tool wear on the fixture radius and the rotation speed test to the Fisher criterion with a confidence probability of $95 \%$ satisfied. On the Figure 4 show the response surface graph depending on the influence of the changed process parameters on the output response.

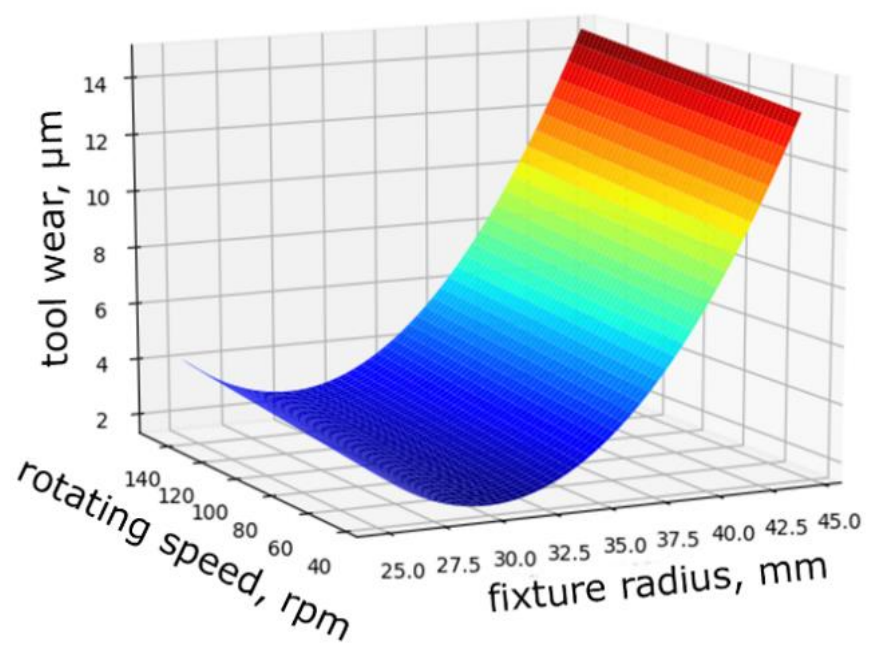

Figure 4. Response surface graph interaction between fixture radius and rotating speed.

The minimal local tool wear was achieved when the workpieces are located in the fixture at the radius of $r=30 \mathrm{~mm}$. However, it increases by 13 times when this value was increased to the maximum value of $44.5 \mathrm{~mm}$ (in this case, the diameter of the fixture covers half the diameter of the lapp).

When the rotation speed increases by more than 3.5 times, the depth of local tool wear increase by $2-3 \%$. The lapp rotation speed has a less significant effect on the local tool wear. Thus, to ensure high productivity, the lapp rotation speed (as well as the cutting speed) should be selected as high as possible.

The detecting algorithm of the processing traces distribution on the lapp surface was designed to determine the nature of tool wear. The point moves according to the specified motion law and intersects the conditional grid on the lapp surface (Figure 5). Each point hit in a specific cell was registered. Accumulation of information about the number of intersections occurred when hits were repeated. Processing traces distribution field on the tool surface is the result of algorithm execution. The wear nature can be determined based on the analysis of the processing traces distribution field (Figure 5).

The software was designed to solve the problem of the uniform distribution of processing traces on the lapp surface. It allows predicting tool wear depending on the setting parameters (the size of the fixture and lapp, the displacement of rotation axes), kinematic parameters (the trajectory type and speed of relative movement), and other parameters such as the material of the workpiece and tool, granularity, concentration, type of excipient, working pressure) of the lapping process. The software was working using the Python programming language on the PyQt5 platform using the Matplotlib library (Figure 6a-c). The results given by the software have been experimentally verified under real processing conditions. The deviation of the geometric tool form was as a result of real processing coincides with the modeled deviation both qualitatively (local wear in the center) and quantitatively (the wear value is $4.5 \mu \mathrm{m}$ ) (Figures 6c and 7). The universal multi-position fixture was designed and manufactured based on the results of a factor experiment and modeling of various setting parameters in the software. The fixture allows you to provide 
mechanized lapping and polishing of the plates ends by sets and increase the production program of finished parts by 12 times. A sustainable of geometric form deviation within $1 \mu \mathrm{m}$ was achieved using the fixture. This is three times more than manual processing.

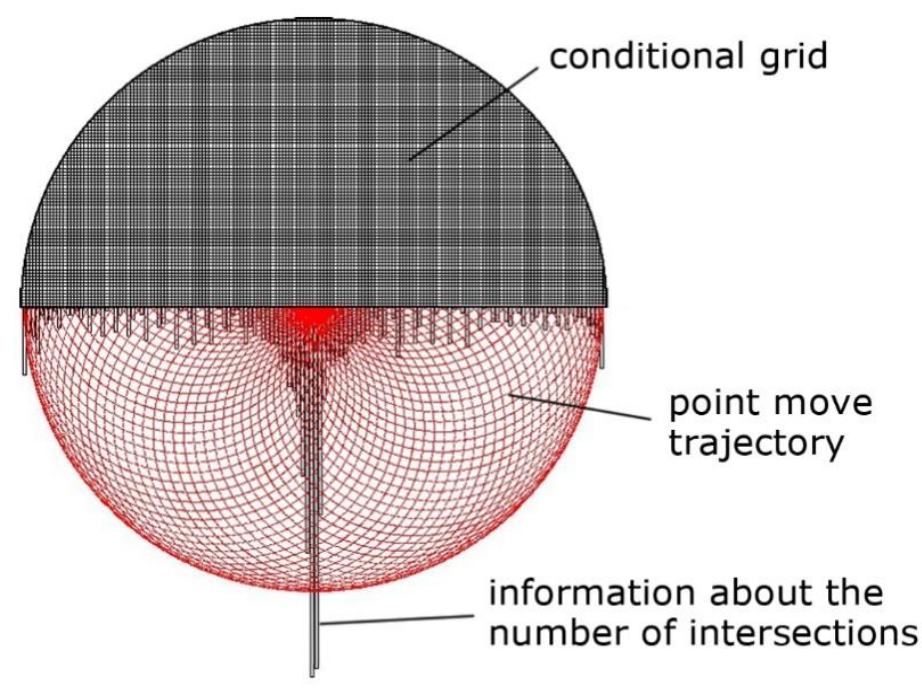

Figure 5. Accumulation of information about the number of intersections in conditional grid cells.

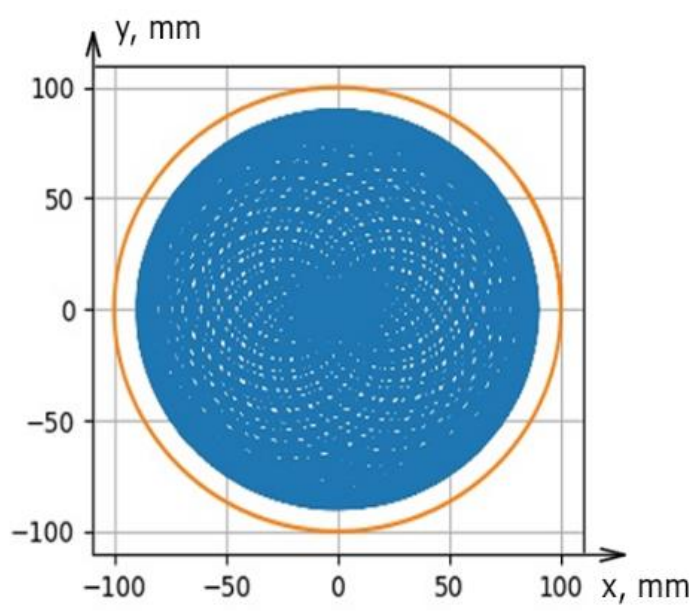

(a)

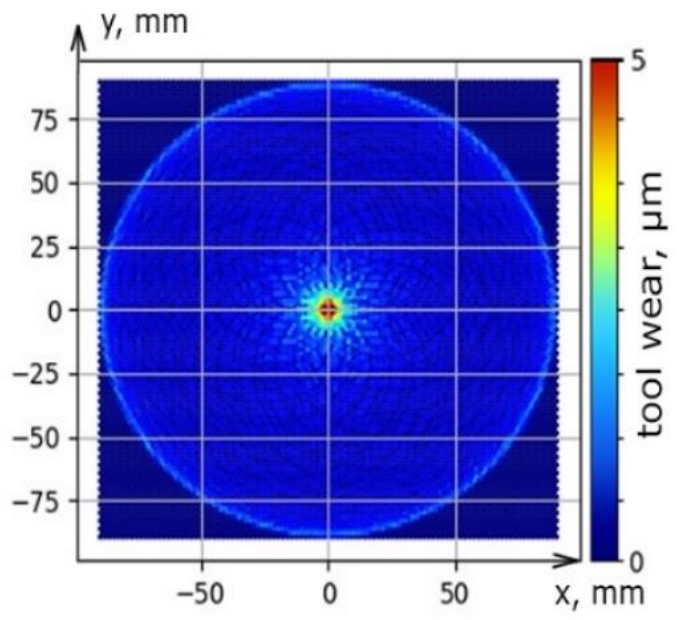

(b)

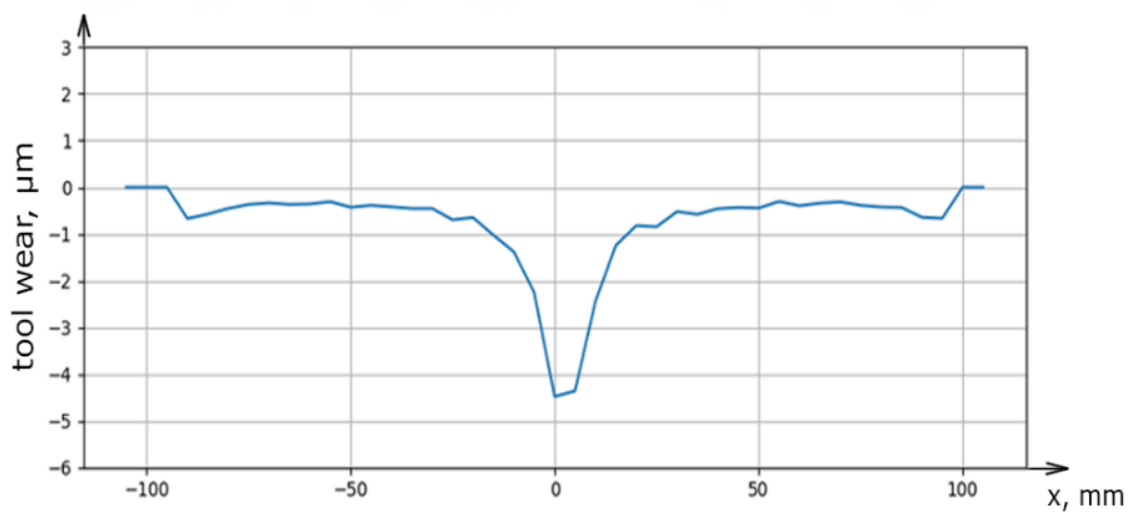

(c)

Figure 6. The result of modeling the trajectory distribution (a) and tool wear (b) on flat and tool wear in the cross-section (c). 


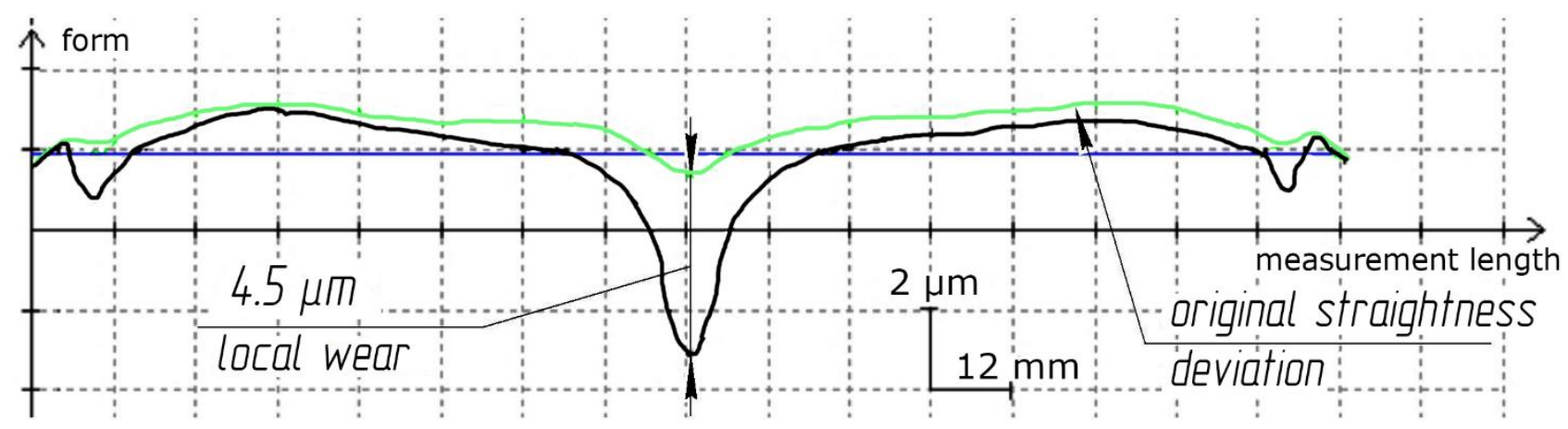

Figure 7. The lapp wear value as a result of real processing conditions.

Plate sets in the fixture were located at a radius of $30 \mathrm{~mm}$ and act as a correct ring, which contributes to more uniform lapp wear. The task of lapping study was to establish the process conditions that ensure a productive removal of the allowance and to obtain a working surface with minimal mechanical defects before the polishing operation. A grain size larger than M14 while maintaining the same concentration leads to the number of abrasive grains decrease in contact with the tool workpiece. It leads to an increase in local forces on individual grains and the appearance of scratches and spalls on the processed surface (Table 2). The removal productivity increases linearly by $2.5-3$ times with increasing pressure (Figure 8a) and cutting speed (Figure $8 b$ ). At the same time, the roughness of the processed surface changes slightly.

Table 2. Influence of abrasive granularity on the lapping process parameters.

\begin{tabular}{cccc}
\hline Abrasive Granularity & Ra, $\mu \mathrm{m}$ & $\begin{array}{c}Q, \\
\mu \mathbf{m} / \mathbf{m i n}\end{array}$ & Technological and Kinematic Lapping Parameters \\
\hline 24A M3-1/5 & 0.079 & 5.09 & Lapp K8, $\mathrm{P}=63,7 \mathrm{kPa}, \mathrm{n}_{\mathrm{lapp}}=96 \mathrm{rpm}$ \\
\hline 24A M7-1/5 & 0.186 & 10.70 & 23.40
\end{tabular}

(a)

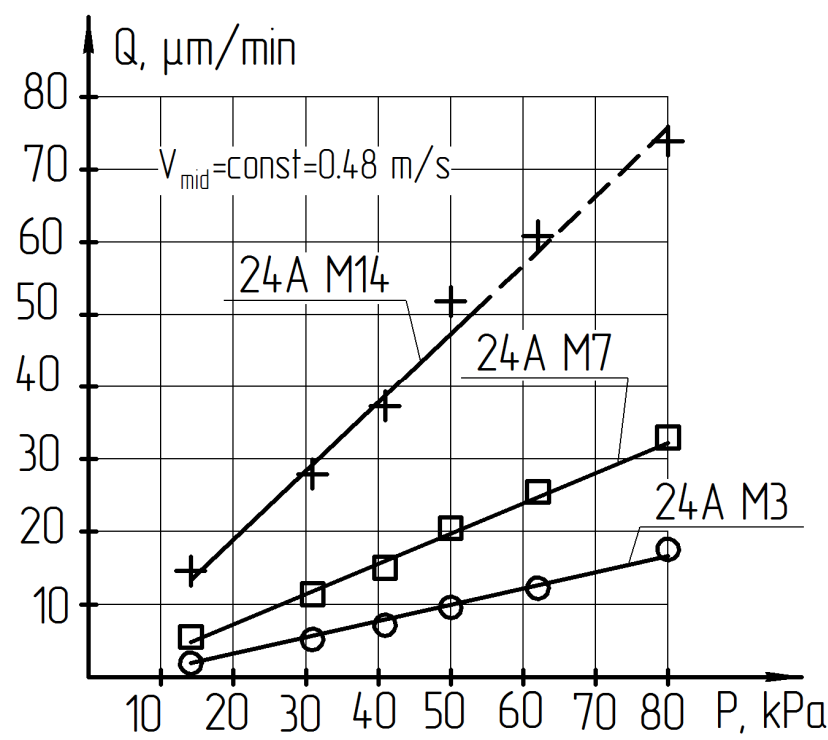

(b)

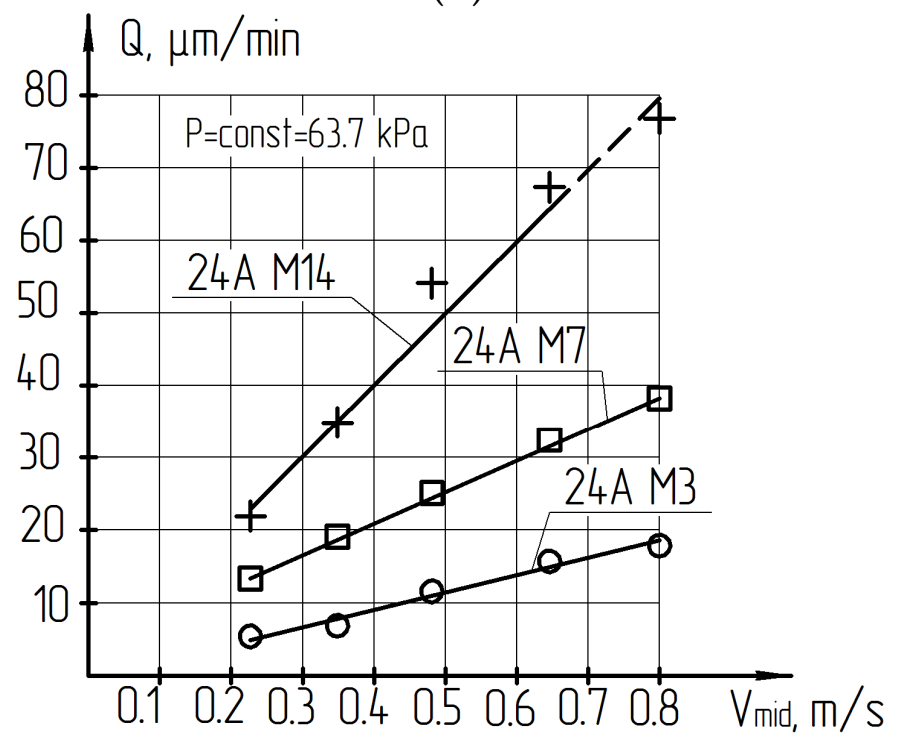

Figure 8. Influence of pressure (a) and mid (mean) cutting speed (b) on the lapping with a free abrasive productivity. 
Deep scratches, cracks and spalls on the processed surface occur when the pressure was exceeded due to the brittleness of the LNSC. In addition, the abrasive suspension was torn from the lapp surface as a result of the action of centrifugal force when the rotation speed increases by more than $120 \mathrm{rpm}$. It can lead to 'dry' friction and analogous defects.

Processing with a fixed abrasive has several advantages compared to lapping with abrasive suspensions. Processing with a fixed abrasive, the productivity and surface roughness were located between the results obtained after lapping with suspensions $24 \mathrm{~A}$ M3 and 24A M7 (Table 3).

Table 3. Results of lapping of LNSC plates with a free and fixed abrasive.

\begin{tabular}{ccccc}
\hline \multirow{2}{*}{ Parameter of the Abrasive Layer } & \multicolumn{3}{c}{ Roughtness, $\boldsymbol{\mu m}$} & \multirow{2}{*}{$\boldsymbol{Q}, \boldsymbol{\mu m} / \mathbf{m i n}$} \\
\cline { 2 - 4 } & $\mathbf{R a}$ & $\mathbf{R z}$ & $\mathbf{R m a x}$ & \\
\hline Suspension 24A M3-1/5 & 0.08 & 0.60 & 0.70 & 5.1 \\
Suspension 24A M7-1/5 & 0.19 & 1.43 & 1.90 & 11.3 \\
Lapp ASN 10/7-B3-01-2 & 0.07 & 0.52 & 0.61 & 10.2 \\
Lapp ASN 10/7-M2-01-2 & 0.13 & 1.02 & 1.28 & 7.8 \\
\hline
\end{tabular}

Analysis of the results showed that the removal productivity increases almost proportionally when the pressure increases three times. The removal productivity increases by 3.5-4 times when the cutting speed increases from 0.22 to $1.1 \mathrm{~m} / \mathrm{s}$. Changes in the pressure and cutting speed do not significantly affect the roughness. In addition, the organic excipient B3-01, which was mainly, contains rubber flour as filler and used for finishing lapping, increases the polishing effect and reduces roughness.

The straightness deviation of the plates ends after lapping on glass lapp of the LK5 mark with $24 \mathrm{~A}$ suspensions with a grain size from 3 to $14 \mu \mathrm{m}$ at a length of $12 \mathrm{~mm}$ did not exceed $0.3-0.5 \mu \mathrm{m}$. The straightness deviation of the plates ends for lapp ASN 10/7-M2-01-2 did not exceed $0.4-0.5 \mu \mathrm{m}$.

The depth of the deformed layer F of the plate's ends of a LNSC was studied by using electron microscopy in the mode of electron backscattering. The SEM of the deformed layer of LNSC after 6 min of lapping on K8 mark glass with 24A suspension, M3, M7, and M14 $\mu \mathrm{m}$ is shown in Figure 9.

(a)

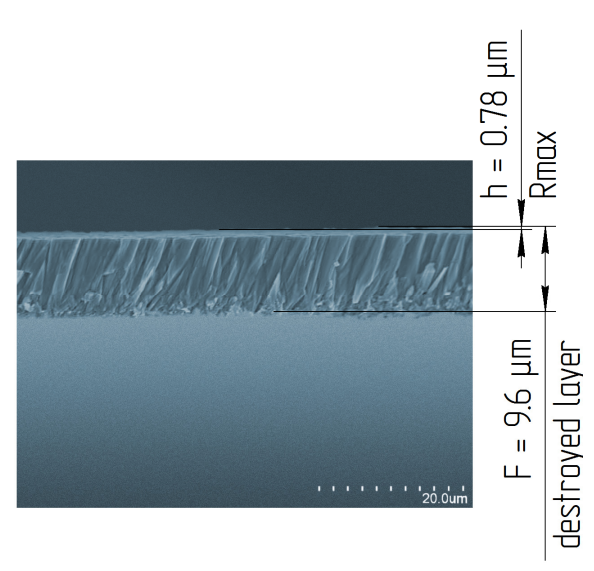

(b)

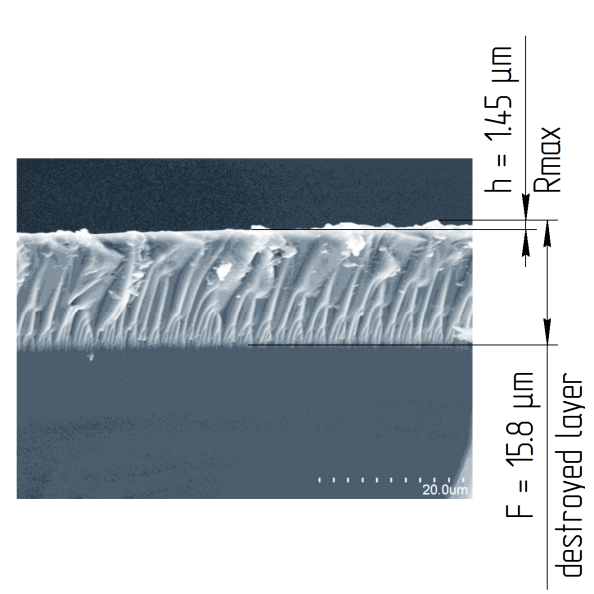

(c)

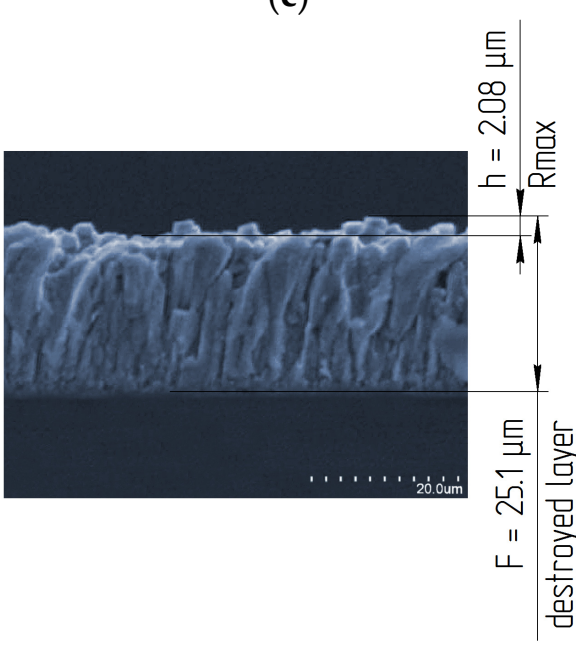

Figure 9. Deformed layer of LNSCplates after lapping on glass lapp of the LK5 mark with 24A suspensions, grain size: (a) M3; (b) M7; (c) M14.

Studies showed that the deformed layer depth $F$ of LNSC is constant during lapping with a specific grain of powder. It confirms the high stability of the lapping process of 
LNSC. Also, studies showed that the structural defects were located at a small angle to the surface. This was due to the predominant direction of lapp movement on the sample.

Figure 10 shows the dependence of the deformed layer depth $F$ on the height of the relief layer $h$. Studies showed that this value correlates with the surface roughness parameter Rmax. Studies of the deformed layer depth Fafter LNSC plate ends lapping were carried out on lapp of optical glass of the K8 mark with a suspension of 24A-1/5 grain size M3, M7, and M14 under the basic processing modes: Vmid $=0.48 \mathrm{~m} / \mathrm{s} ; \mathrm{P}=31 \mathrm{kPa}$.

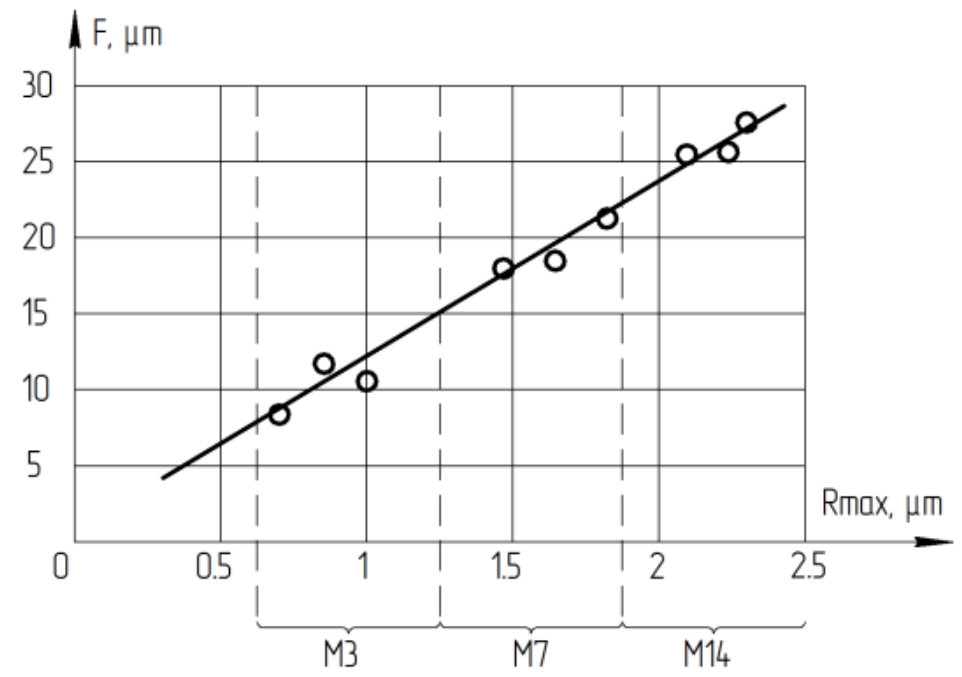

Figure 10. Dependence of the deformed layer depth on the height of the relief layer $h$ (Rmax).

The obtained dependence allows us to determine the value of the ratio of the deformed layer depth $F$ and the height of the relief layer $h$. This ratio is $F / h=12$ in the crystallographic direction during lapping of the LNSC plates ends. This conclusion allows us to estimate the deformed layer value by rapid measurements of the processed surface roughness (Rmax) of LNSC using a portable profilometer in the production environment. This makes it possible to exclude studies on expensive time consuming equipment such as electron microscopy. The ratio of the deformed and relief layers during processing with a fixed abrasive was determined in the same way. During processing on the ASN 10/7-B3-01-2 lapp, the ratio is also 1:12. Deformed by lapping layer requires removal during the subsequent polishing operation of the single crystal. The formula was recommended to calculate the total deformed lapping layer depth $(\mathrm{F}): \mathrm{F}=12 \cdot \mathrm{Rmax}, \mu \mathrm{m}$. The presented results was in line with the conclusions drawn by the authors $[3-5,7,9]$.

The purpose of polishing was to remove the material that exceeds the size of the deformed layer after the lapping operation and to obtain a surface free from mechanical defects. The results of comparative experiments on polishing productivity and surface quality are shown in Table 4 . The polishing process durability was estimated by the total machine operating time $\Sigma t_{\text {mach }}$ before the appearance of local centers of physical wear on the work surface or before a significant decrease in the polishing ability and the appearance of visible scratches on the polished surface.

Table 4 shows that synthetic woven hydrophobic materials such as raincoat fabrics, and non-woven polishers Microlap and VelTex, showed the highest removal productivity. However, woven material polishers are slightly worse than non-woven ones in roughness parameters. The higher elasticity and uniformity of the structure of non-woven materials provides a more uniform grain appearance in the abrasive layer, and good 'adaptability' of polishers to the processing surface. 
Table 4. Polisher material influence on the indicators of the polishing process.

\begin{tabular}{|c|c|c|c|c|c|c|}
\hline & \multirow{2}{*}{ Polisher Material } & \multicolumn{3}{|c|}{ Roughness, $\mu \mathrm{m}$} & \multirow{2}{*}{$Q, \mu \mathrm{m} / \mathrm{min}$} & \multirow{2}{*}{ Durability $\Sigma t_{\text {mach }}, \min$} \\
\hline & & $\mathbf{R a}$ & $\mathbf{R z}$ & Rmax & & \\
\hline \multirow{4}{*}{ Woven } & Raincoat fabric (Bologna) & 0.004 & 0.014 & 0.017 & 3,23 & \multirow{3}{*}{$\sim 40-50$} \\
\hline & $\begin{array}{c}\text { Raincoat fabric ( } 80 \% \text { polyamide, } \\
20 \% \text { polyurethane) }\end{array}$ & 0.002 & 0.011 & 0.013 & 2.97 & \\
\hline & $\begin{array}{l}\text { Awning synthetic fabric with a } \\
\text { water-repellent coating }\end{array}$ & 0.002 & 0.013 & 0.014 & 3.17 & \\
\hline & $\begin{array}{l}\text { Nylon mesh with a thickness of } \\
0.09 \mathrm{~mm} \text {, cell size } \sim 0.1 \mathrm{~mm}\end{array}$ & 0.001 & 0.010 & 0.012 & 0.61 & $\sim 6-10$ \\
\hline \multirow{3}{*}{ Non-woven } & $\begin{array}{l}\text { Polyethylene wrap with a } \\
\text { thickness of } 0.14 \mathrm{~mm}\end{array}$ & 0.001 & 0.009 & 0.011 & 1.78 & $\sim 6-10$ \\
\hline & Synthetic leather SK-8 & 0.001 & 0.005 & 0.007 & 1.12 & \multirow{2}{*}{ more 120} \\
\hline & $\begin{array}{l}\text { MicrolapRemet and VelTex } \\
\text { Buehler polisher }\end{array}$ & 0.001 & 0.006 & 0.006 & 2.91 & \\
\hline
\end{tabular}

Comparing the total machine operating time $\Sigma t_{\text {mach }}$ (Table 4) shows that during the processing of plates ends surface with a small contact area, the durability of the polisher is determined not only by the strength of the material but also by its structure. As a result, of the impact of sharp edges of the processed plates, the fibers of polishers are deformed; local breaks and cuts appear on the surface of the polisher. Non-woven polishers with a fleecy structure (synthetic leather SK-8, MicrolabRemet and VelTex Buehler polishers) showed the highest durability with a diamond suspension grain size of $1 / 0.5$ and a concentration of $1 / 10$ in the basic modes. During polishing on thicker and softer (fleecy) polishers (raincoat fabric, Microlap, or VelTex), reducing the suspension granularity to $0.5 / 0$ and significantly reduces the speed of polishing with a simultaneous decline in surface quality (defects in the form of holes and swells).

The polishing productivity was almost directly proportional to the average cutting speed. A four-fold increase in speed has almost no effect on the polished surface roughness. The speed limit was the same as for lapping-the risk of 'dry' friction and the durability of the polisher.

The purpose of the polishing operation was to create the required roughness and remove the layer deformed by lapping. Figure 11 shows that the required roughness is set after 2-3 min of processing. However, the deformed layer will not be completely removed during this time. The minimum polishing time was calculated based on the removal rate during polishing, the maximum depth $\mathrm{h}$ of the relief layer (Rmax), and the ratio of the depth of the deformed $\mathrm{F}$ for a LNSC was 1:12.

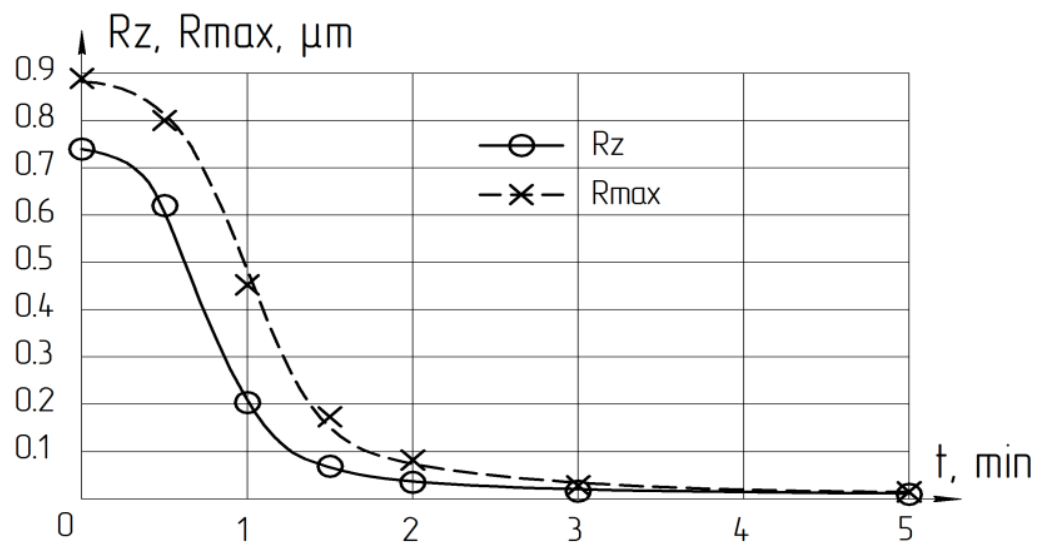

Figure 11. Polishing cycle time influence on the surface roughness of LNSC. 
So, during the abrasive finish processing of crystalline materials (plate's end of LNSC) the total depth of the deformed layer by lapping and the minimum polishing time required for its removal was calculated. The formula recommended is $F=12 \cdot \mathrm{Rmax}, \mu \mathrm{m}$; $T_{\text {pol }} \geq F / Q_{\text {pol }}$, min.

In the production environment, the calculation of the minimum polishing cycle time allowed to increase the processing efficiency by reducing the time of the polishing operation, reducing the number of polishing defects, and reducing the abrasive materials consumption. The presented results coincided with the authors' conclusions [3-5,11-14].

\section{Conclusions}

1. The tool movement kinematics have shown that for lapping and polishing of brittle materials such as lithium niobate single crystal, it is advisable to use machines with a rotational movement. Machines with oscillating or translational tool movement are not recommended for plates ends finishing operations due to the high rate of change in the speed and acceleration vector direction.

2. The minimal local tool wear is achieved when the workpieces are located in the fixture at the radius of $r=30 \mathrm{~mm}$; however, increases by 13 times when this value is increased to the value of $44.5 \mathrm{~mm}$. When the rotation speednincreases by more than 3.5 times, the depth of local tool wear increases by $2-3 \%$. Therefore, to ensure high productivity, the lapp rotation speed (as well as the cutting speed) should be selected as much as possible.

3. The designed software allows to prediction tool wear depending on the setting parameters (the size of the fixture and lapp, the displacement of rotation axes), kinematic parameters (the trajectory type and speed of relative movement), and technological parameters (the material of the workpiece and tool, granularity, concentration, type of excipient, working pressure) of the lapping process.

4. The plates lapping with free abrasive results have shown that the removal productivity increases linearly with increasing pressure and cutting speed. The roughness of the processed surface changes slightly. Acceptable pressure values depend on the type and grain size of the abrasive and the suspension concentration. While using a $24 \mathrm{~A}$ M14 suspension of one-fifth concentration, the permissible pressure is not more than $40 \mathrm{kPa}$. While using 24A M7 and 24A M3 suspensions of the same concentration, the pressure can be increased to $80 \mathrm{kPa}$. Increasing the abrasive grain size larger than $\mathrm{M} 14 \mu \mathrm{m}$ is impractical. The recommended lapp rotation speed should not exceed $120 \mathrm{rpm}$.

5. For processing brittle materials, such as lithium niobate single crystal, it is possible to use the lapping method with fixed abrasive, having significant advantages, on diamond lapp ASN10/7-B3-01-2.

6. Studies have shown that during lapping of LNSC plates, a deformed layer of material is formed on the surface layer as a result of the impact of abrasive grains. This layer must be removed during the subsequent polishing operation. The deformed layer depthFis determined by the ratio: $\mathrm{F}=12 \cdot \mathrm{h}$, wherehis the relief layer height. The relief layer height is characterized by the Rmax of the processed surface roughness. Rmax can be rapidly measured by a portable profiler in a production environment.

7. The effectiveness of polishing process using Microlab Reset and VelTex Buehler nonwoven polishers and SK-8 artificial leather has been proven. The diamond polishing suspension granularity lower limit is determined to be $1 / 0.5 \mu \mathrm{m}$. The processing modes are recommended: polisher rotation speed is $180-220 \mathrm{rpm}$ and the pressure is 80-100 kPa, which ensures the required quality and high processing productivity.

8. The formula recommended calculating the total depth of the deformed layer by lapping and the minimum polishing time required for its removal: $F=12 \cdot \operatorname{Rmax}, \mu \mathrm{m}$; Tpol $\geq$ F/Qpol., min. 
Author Contributions: Conceptualization, K.R.M., T.R.A. and E.S.S.; Methodology, E.A.G. and S.S.S.; Software, E.A.G. and T.R.A.; Validation, K.R.M., E.S.S. and T.R.A.; Formal analysis, K.R.M., E.S.S. and E.A.G.; Investigation, K.R.M., S.S.S. and T.R.A.; Validation, E.A.G., E.S.S. and S.S.S.; Resources, K.R.M. and E.S.S.; Data curation, K.R.M. and E.S.S.; Writing-original draft preparation, E.A.G. and T.R.A.; Writing-review and editing, E.S.S., E.A.G., S.S.S. and T.R.A.; Visualization, T.R.A.; Supervision, K.R.M. and T.R.A.; Project administration, T.R.A.; Funding acquisition, T.R.A. and K.R.M. All authors have read and agreed to the published version of the manuscript.

Funding: The research was funded from Russian Ministry of Science and Higher Education (project FSNM-2020-0028).

Institutional Review Board Statement: Not applicable.

Informed Consent Statement: Not applicable.

Data Availability Statement: The authors have all the data and materials of the study.

Conflicts of Interest: The authors declare no conflict of interest. The funders had no role in the design of the study; in the collection, analyses, or interpretation of data; in the writing of the manuscript, or in the decision to publish the results.

\section{References}

1. Nannan, Z.; Hong-Jun, W.; Dao-Chun, H.; Xiu-Juan, W. Research of Subsurface Damage Depth of Lithium Niobate Crystal by Fixed-Abrasive Lapping. Integr. Ferroelectr. 2020, 209, 181-187. [CrossRef]

2. Guo, L.; Zhang, X.; Chen, S.; Hui, J. An Experimental Study on the Precision Abrasive Machining Process of Hard and Brittle Materials with Ultraviolet-Resin Bond Diamond Abrasive Tools. Materials 2019, 12, 125. [CrossRef] [PubMed]

3. Yu, I.; Golovin, C.H.; Dub, V.I.; Ivolgin, B.B.; Korenkov, A.I.; Tyurin. Kineticheskieosobennostideformaciitverdyhtel v nano- I mikroobemah. Fiz. Tverd. 2005, 47, 961-973.

4. Li, X.; Kasai, T.; Nakao, S.; Ando, T.; Shikida, M.; Sato, K.; Tanaka, H. Measurement for fracture toughness of single crystal silicon film with tensile test. Sens. Actuators 2005, 119, 229-235. [CrossRef]

5. Kim, H.M.; Manivannan, R.; Moon, D.J.; Xiong, H.; Park, J.G. Evaluation of double sided lapping using a fixed abrasive pad for sapphire substrates. Mater. Res. Soc. Proc. 2013, 302, 1340-1344. [CrossRef]

6. Preston, F.W. Journ. Soc. Glass Technol. 1933, 17, 5.

7. Venkatesh, V.C.; Izman, S.; Sharif, S.; Mon, T.T.; Konneh, M. Ductile streaks in precision grinding of hard and brittle materials. Sadhana 2003, 28, 915-924. [CrossRef]

8. Natsu, W.; Ito, Y.; Kunieda, M.; Naoi, K.; Iguchi, N. Effect of support method and mechanical property of $300 \mathrm{~mm}$ silicon wafers on sori measurement. Precis. Eng. 2005, 29, 19-26. [CrossRef]

9. Hanheng, D.; Yip, S.Z.; Zhu, Z.; Sandy, T. Ultra-Precision Diamond Machined Free form Optical Parts and Structures. J. Manuf. Sci. Eng. 2018, 142, 65-97.

10. Okatova, M.A. Spravochnikoptikatekhnologa. Politekhnika 2004, 654-679.

11. Artyomov, A.S. Nanoalmazydlyapolirovaniya. Fiz. TvyordogoTela 2004, 46, 89-97.

12. Tam, H.Y.; Cheng, H.B.; Wang, Y.W. Removal rate and surface roughness in the lapping and polishing of RB-SiC optical components. J. Mater. Process. Technol. 2007, 192-193, 276-280. [CrossRef]

13. Kuznecov, S.M. Ob uprochneniipoverhnostnogosloyastekla. Stekloikeramika 1970, 3, 11-13.

14. Jiao, Y.; Zhen, S.; Li, S.; Wang, X.; Bo, F.; Xu, J.; Zhang, G. Nano-Domains Produced through a Two-Step Poling Technique in Lithium Niobate on Insulators. Materials 2020, 13, 3617. [CrossRef] [PubMed]

15. De Payrebrune, K.M. Relation of kinematics and contact forces in three-body systems with a limited number of particles. Facta Univ. Ser. Mech. Eng. 2021, 1, 14.

16. Alejnikov, F.K. Finishnyeoperacii. ZhurnalTekhnicheskojFiz. 1957, 27, 2725.

17. Kukleva, Z.A. Onekotoryhosobennostyahprocessashlifovaniyakristallov. Trudy GOI 1960, 28, 98-104. 